\title{
63. Zur Theorie der algebraischen Korrespondenzen II. Multiplikation der Korrespondenzen.
}

\author{
Von Kenkiti Iwasawa. \\ Mathematisehes Institut, Kaiserliche Jniversität zu Tokio. \\ (Comm. by T. TAKAGI, M.I.A., Nov. 12, 1945.)
}

In dieser Note wollen wir die Multiplikation der algèbraischen Korrespondenzen auf algebraischen Kurven definieren und einige Sätze darüber herleiten. Der Multiplikatorenmodul und der Multiplikatorenring der algebraischen Kurve sollen auch eingeführt werden. Die Bezeichnungen wie $k, P_{l}, P_{m}, P_{l, m}, \Gamma_{1}, \Gamma_{2}$, $I_{12}^{\prime}$ u.s.w. mögen dieselbe Bedeutungen haben wie $\mathrm{Im}$ Teil $\mathrm{I}^{1)}$, wenn nicht das. Gegenteilige angemerkt wird.

Zunächst führen wir noch einige Bezeichnungen ein. Für eine irreduzible Korrespondenz $C$ auf $\Gamma_{\text {t2 }}$ und einen beliebigen Punkt $a$ in $\Gamma_{1}$ definieren wir eine Punktgruppe $C^{(2)}(a)$ in $\Gamma_{2}$ mit

$$
\begin{aligned}
& C^{(a)}(a)=\left\{b^{(\mu)} ;\left(a, b^{\mu}\right) \in\left[(a) \times \Gamma_{2}, C\right]\right\},{ }^{2)} \quad \text { falls } C \neq(a) \times \Gamma_{2}, \\
& C^{(2)}(a)=\text { die Nullgruppe, falls } C=(a) \times \Gamma_{2} .
\end{aligned}
$$

Allgemein setze man für eine beliebige Korrespondenz $C=\Sigma \lambda_{i} C_{i}\left(C_{i}\right.$ : irreduzibel) auf $\Gamma_{12}$ und eine beliebige Punktgruppe $G_{1}=\Sigma \mu_{j} a^{(j)}$ in $\Gamma_{1}$

$$
C^{(2)}\left(G_{1}\right)=\Sigma \lambda_{i} \mu_{j} C_{i}^{(3)}\left(a^{(D)}\right) \text {. }
$$

Analog definieren wir $C^{(1)}(b)$ bzw. $C^{(1)}\left(G_{2}\right)$ für einen Punkt $b$ bzw. eine Punktgruppe $G_{2}$ in $\Gamma_{2}$, Es gilt dann offenbar

$$
C^{(2)}\left(G_{11}\right) \rightarrow C^{(2)}\left(G_{1}^{\prime}\right), C^{(1)}\left(G_{2}\right) \rightarrow \Theta^{(1)}\left(G_{2}^{\prime}\right) \text {, }
$$

wenn $\operatorname{man} G_{i}(i=1,2)$ in $G_{i}^{i}$ spezialisiert.

Nun beweisen wir den

Satz 1. Es gilt fụur jeden Punkt $a$ in $\Gamma_{1}$

$$
C^{(2)}(a)=D^{(2)}(a)
$$

genau dann, wenth die Korrespondenzen $C, D$ bis auf Korrespondenzen der Form $\left(a^{(i)}\right) \times \Gamma_{\mathrm{a}}$ mit einander zusammenfallen. Analoges gilt für $C^{(1)}, D^{(1)}$.

Beweis. Es sei

$$
C=\Sigma \lambda_{i} C_{i}, D=\Sigma \mu_{j} D_{j}
$$

und es gelte für einen allgemeinen Punkt $\xi$ von $\Gamma_{1}$

$$
\Sigma \lambda_{i} C_{i}^{(2)}(\xi)=\Sigma \mu_{j} D_{j}^{(-)}(\dot{\xi})
$$

1) Zur Theorie der algebraischen Korrespondenzen I, Proc. Imp. Acad. Jap. Vol. (1945), zitiert im folgenden mit I.

2) $[C, D]$ bedeutet die Schnittpunktgruppe voin C, D, vgl. I. $(\alpha ; E)$ bedeutet die Menge von $\alpha$, welches die Eigenschaft $\mathbf{E}$ besitzt. 
Wonn $C_{i}$ nicht die Form $\left(a^{(i)}\right) \times \Gamma_{2}$ hat, so wird $C_{i}$ nach Satz 3 in I durch $C_{i}^{(2)}(\xi)=\left[(\xi) \times \Gamma_{z}^{\prime}, C_{i}\right]$ eindeutig bestimmt. Dasselbe gilt natürlich auch für $D_{j}$ und daraus folgt offenbar die Behauptung.

Nun sei $\Gamma_{s}$ eine singularitätenfreie irreduzible Kurve in einem n-dimensionalen projektiven Raum $P_{n}$ über $k$. Wir setzen

$$
\begin{gathered}
P_{l, n}=P_{l} \times P_{n}, P_{m, 2}=P_{m} \times P_{u,}, \\
\Gamma_{1,3}=\Gamma_{1} \times \Gamma_{*}, \Gamma_{2,3}=\Gamma_{2} \times \Gamma_{3 \bullet}
\end{gathered}
$$

Für eine beliebige Korrespondenz $C=C_{12}$ auf $\Gamma_{12}$ und ein $D=D_{z_{3}}$. auf $\Gamma_{23}$ wollen wir eine solche Korrespondenz $E=E_{13}$ auf $\Gamma_{13}$ suchen, so dass as für beliebige Punkte $a, c$ in $\Gamma_{1}$ bzw. $\Gamma_{\mathrm{s}}$ immer

$$
E_{13}^{(3)}(a)=D_{23}^{(3)}\left(C_{12}^{(2)}(a)\right), E_{13}^{(1)}(c)=C_{12}^{(1)}\left(D_{33}^{(2)}(c)\right)
$$

gilt. Es giht, wie wir zeigen werden, eine einzige solche Korrespondenz $E_{13}$ und wir heissen diese Korrespondenz das Produkt von $C$ und $D$ :

$$
\mathrm{E}_{13}=\mathrm{C}_{12} \times \mathrm{D}_{23}
$$

Die Eindeutigkeit folgt ohne Weiters aus Satz 1. Beim Beweis der Existenz kann man $C, D$ als irreduzibel annehmen; denn für allgemeine $\mathrm{C}=\Sigma \lambda_{i} C_{i}, D=$ $\boldsymbol{\Sigma}_{\mu_{j}} D_{j}$ hat man nur zu setzen

$$
E=C \times D=\Sigma \lambda_{i} \mu_{i}\left(C_{i} \times D_{j}\right),
$$

wenn $C_{j} \times D_{j}$ schon bestimmt ist. Wenn eines von $\mathrm{C}, \mathrm{D}$ ausgeartet ist, so können wir ohne Weiters ein solches $E$ angeben. Z.B. setze man für $C=\Gamma_{1} \times(b)$

$$
E=C \times D=\Sigma\left(\Gamma_{1} \times\left(c^{(j)}\right)\right),
$$

wobei $c^{(j)}$ alle Punkte in $D^{(3)}(b)$ durchläuft. Man bemerke dabei, dass $E$ in diesem Fall immer nur aus ausgearteten irroduziblen Kórrespondenzen besteht und dass in gewissen Fallen die Nullkorrespondenz $\theta_{13}$ auftritt; es gilt z.B.

$$
\left((a) \times \Gamma_{2}\right) \times\left(\Gamma_{2} \times(c)\right)=\theta_{13} .
$$

Wir nehmen also an, dass $C, D$ beide irreduzible nicht ausgeartete Korrespondenzen sind und beweisen die Existenz von $E=C \times D$. Wir bezeichnen mit $f(x, y, u)=0$ diejenige Hyperfläche in $P_{l},{ }_{m}$, die $C$ mit einem allgemeinen linearen Raum $L_{l-2}^{(1)} \times L_{m-2}^{(2)}$ verbindet und mit $g\left(u, z, v^{\prime}\right)^{3)}=0$ die Hyperfläche in $P_{m},{ }_{n}$, die $D$ mit einem ebensolchen Raum $L_{m-2}^{\prime(2)} \times L_{n-2}^{\prime(3)}$ verbindet. Es gilt dann

$$
\left[\Gamma_{1:}, f\left(x, y, u^{\prime}\right)\right]=C+\sum_{i=2}^{a} \lambda_{i} C_{i},\left[\Gamma_{23}, g\left(x, y, v^{\prime}\right)\right]=D+\sum_{j=2}^{\beta} \mu_{j} D_{j},
$$

wobei $C_{i}(i \geqq 2), D_{j}(j \geqq 2)$ je von $L_{2-2}^{(1)} \times L_{m-2}^{(2)}$ bzw. $L_{m-2} \times L_{m-2}^{\prime(3)}$ abhängige irreduzible Korrespondenzen sind. ${ }^{4)}$ Wir schneiden nun in $P_{m}$ die $\mathrm{Hry}_{\mathrm{v}}$ erflächen

3) Vgl. I. Wir bezeichnen die Unbestimmten in $P_{i 2}$ mit $z=\left(z_{0}, z_{1}, \ldots, z_{i 2}\right)$.

4) Vgl. I. 
$f\left(x, y, u^{\prime}\right)=0, g\left(y, \cdot z, v^{\prime}\right)=0$, wobei $f, g$ als Form von $y$ betrachtet werden sollen, mit der Kurve $\Gamma_{2}^{\prime}$. $\quad$ Es sei

$$
\begin{aligned}
& {\left[\Gamma_{2}, f\left(x, y, u^{\prime}\right)\right]=\left\{\eta^{(\lambda)} ; \lambda=1, \ldots, \gamma\right\},} \\
& {\left[\Gamma_{2}, g\left(y, z, v^{\prime}\right)\right]=\left\{\eta^{\prime(\mu)} ; \mu=1, \ldots, \delta\right\} .}
\end{aligned}
$$

Mit normierten Koordinaten gilt dann ${ }^{5}$

$$
\prod_{\mu=1}^{\delta} f\left(x, \eta^{(\mu)}, u^{\prime}\right)=\stackrel{\Gamma}{\lambda=1}_{\lambda=1} g\left(\eta^{(\lambda)}, z, v^{\prime}\right)
$$

und dies ist eine Form von $x, z$, welche wir mit $h\left(x, z, w^{\prime}\right)$ bezeichnen wollen. Es sei $E^{\prime}$ der von $L_{l-2}^{(1)} \times L_{m-2}^{(2)}, L_{m-2}^{(2)} \times L_{n-2}^{\prime(3)}$ unabhängige Teil von $\left[\Gamma_{13}, h(x, z\right.$, $\left.w^{\prime}\right)$ ] und $E^{\prime}$ der Rest:

$$
\left[\Gamma_{1:}, h\left(x, z, w^{\prime}\right)\right]=E^{\prime}+E^{\prime \prime} .
$$

Wir wollen nun aus der Annahme, dass $\left[\Gamma_{1 s}, h\left(x, z, w^{\prime}\right)\right]$ eine ausgeartete irreduzible Korrespondenz enthält, einen Widerspruch abjeiten. . Es sei also z.B. $\left(a^{\prime}\right) \times \Gamma_{\mathrm{3}}$ in $\left[\Gamma_{12}, l l\left(x, z, w^{\prime}\right)\right]$ enthalten und es gelte $\eta^{(\lambda)} \rightarrow \bar{\eta}^{(\lambda)}$ bei der Spezialisierung $x \rightarrow a^{\prime}$. Es gilt dann

$$
h\left(a^{\prime}, z, w^{\prime}\right)=\Pi g\left(\eta^{(\lambda)}, z, w^{\prime}\right)
$$

und eines von $g\left(\bar{\eta}^{(\lambda)}, z, v^{\prime}\right)=0$ muss $\Gamma_{3}$ enthalten, d.h. eines von $\left(\bar{\eta}^{(\lambda)} \times \Gamma_{3}\right)$ muss in $\left[\Gamma_{23}, g\left(y, z, v^{\prime}\right)\right]$ liegen, was aber unmöglich ist, wie wir in $I$ bemerkt haben. $\left[\Gamma_{1:}, h\left(x, z, w^{\prime}\right)\right]=E^{\prime}+E^{\prime \prime}$ enthält also keine ausgeartete Korrespondenz und folglich wird nach Satz 1 durch das Bild $E^{\prime(3)}(\xi)+E^{\prime \prime(3)}(\xi)$ von cinem allgemeinen Punkt $\xi$ von $\Gamma_{1}$ völlig bestimmt. $E^{\prime(3)}(\xi)$ ist duhei offenbar von $L_{l-1}^{(1)} \times L_{m-2}^{(2)}, L_{m-2}^{\prime(2)} \times L_{n-2}^{\prime(3)}$ unabhängig, während jeder Punkt von $E^{\prime \prime(3)}(\xi)$ wirklick von einem solcher Räume abhäingt. Wenn nun $\eta^{(\lambda)}$ bei der Spezialisicrung $x \rightarrow \xi$ relationstreu in $\eta^{*(\lambda)}$ übergeht, so ist

$$
\left[\Gamma_{2}, f\left(\xi, y, u^{\prime}\right)\right]=\left\{\eta^{*(\lambda)} ; \lambda=1, \ldots \gamma\right\}, h\left(\xi, z, w^{\prime}\right)=I I g\left(\eta^{*(\lambda)}, z, v^{\prime}\right) .
$$

Es gilt andererseits nach Satz 4 in 1

$$
\begin{gathered}
E^{\prime(3)}(\xi)+E^{\prime \prime(3)}(\xi)=\left[\Gamma_{13}, h\left(x, z, w^{\prime}\right)\right]^{(3)}(\xi)=\left\{\zeta^{(\omega)} ;\left(\xi, \zeta^{(\omega)}\right) \epsilon\left[(\xi) \times \Gamma_{: 3,},\right.\right. \\
\left.\left.h\left(x, z, w^{\prime}\right)\right]\right\}=\left[\Gamma_{3}, h\left(\xi, z, w^{\prime}\right)\right],
\end{gathered}
$$

ebenso

$$
\begin{aligned}
{\left[\Gamma_{23}, g\left(y, z, v^{\prime}\right)\right]^{(3)}\left(\eta^{*(\lambda)}\right) } & =\left[\Gamma_{3}, g\left(\eta^{*(\lambda)}, z, v^{\prime}\right)\right], \\
{\left[\Gamma_{12}, f\left(x, y, u^{\prime}\right)\right]^{(2)}(\xi) } & =\left[\Gamma_{2}, f\left(\xi, y, u^{\prime}\right)\right] .
\end{aligned}
$$

Es folgt also

$$
E^{\prime(3)}(\xi)+E^{\prime \prime(3)}(\xi)=\left[\Gamma_{3}, h\left(\xi, z, u^{\prime}\right)\right]=\left[\Gamma_{:}, \stackrel{I}{i=1}_{I} g\left(\eta^{*(\lambda)}, z, v^{\prime}\right)\right]
$$

5) Vgl. W.-L. Chow, Die geometrische 'Theorie der algebraischen Funktionen für beliebige vollkommene Körper, Math. Ann. 114 (1937). 


$$
\begin{aligned}
& =\sum_{\lambda=1}^{\Upsilon}\left[\Gamma_{i}, g\left(\eta^{*(\lambda)}, z, v^{\prime}\right)\right]=\sum_{\lambda=1}^{\Upsilon}\left[\Gamma, \ldots, g\left(y, z, v^{\prime}\right)\right]^{(3)}\left(\eta^{*(\lambda)}\right) \\
& =\left[\Gamma_{2 \xi ;}, g\left(y, z, v^{\prime}\right)\right]^{(3)}\left(\sum_{\lambda=1}^{\gamma} \eta^{*(\lambda)}\right) \\
& =\left[\Gamma_{2}, g\left(y, z, v^{\prime}\right)\right]^{(3)}\left(\left[\Gamma_{2,} f\left(\xi, y, u^{\prime}\right)\right]\right) \\
& =\left[\Gamma_{2:}, g\left(y, z, v^{\prime}\right)\right]^{(3)}\left(\left[\Gamma_{12}^{\prime}, f\left(x, y, u^{\prime}\right)\right]^{(2)}(\xi)\right) \\
& =\left(D+\sum_{j=2}^{\beta} \eta_{j} D_{j}\right)^{(3)}\left(\left(C+\sum_{i=2}^{\alpha} \lambda_{i} C_{i}\right)^{(3)}(\xi)\right) \text {. }
\end{aligned}
$$

Mithin haben wir

$$
\text { (3) } \begin{aligned}
E^{\gamma(3)}(\xi) & +E^{\prime \prime(3)}(\xi)=D^{(3)}\left(C^{(2)}(\xi)\right)+\sum_{i=2}^{\alpha} \alpha_{i} D^{(3)}\left(C_{i}^{(2)}(\xi)\right)+\sum_{j=2}^{\beta} \mu_{j} D_{j}^{(3)}\left(C^{(2)}(\xi)\right) \\
& +\sum_{i=2 j=2}^{\alpha \beta} \sum_{i} \lambda_{i} \mu_{j} D_{j}^{(3)}\left(C_{i}^{(2)}(\xi)\right) .
\end{aligned}
$$

Offenbar hängen díe Funkte in $D^{(3)}\left(C^{(2)}(\xi)\right)$ nicht von $L_{l-2}^{(1)} \times L_{m-2}^{(2)}, L_{m-2}^{\prime(2)} \times L_{n-2}^{\prime(3)}$ ab. Wir zeigen num, dass jeder Punkt von $D^{(3)}\left(C_{i}^{(2)}(\xi)\right), D_{j}^{(3)}\left(C^{(2)}(\xi)\right), D_{i}^{(3)}\left(C_{i}^{(2)}(\xi)\right)$ von einem solcher linearen Räume wirklỉch abhängt. Z.B. nehme man einem Punkt $\zeta^{(\omega)}$ in $D^{(3)}\left(C_{i}^{(2)}(\xi)\right)$. Nach Konstruktion gibt es einem Punkt $\eta^{*(\lambda)}$, so dass $\left(\xi, \eta^{*(\lambda)}\right)$ ein allgemeiner Punkt von $C_{i}$ und $\left(\eta^{*(\lambda)}, \zeta^{(\omega)}\right)$ ein allgemeiner Punkt von $D$ ist. $\left(\xi, \eta^{*(\lambda)}\right)$ und $\eta^{*(\lambda)}$ hängen also nicht von $L_{m \mid 2}^{\prime(2)} \times L_{n-2}^{\prime(3)}$ ab, während $\left(\eta^{*(\lambda)}, \zeta^{(\omega)}\right)$ wirklich von diesem Raum abhängt. $\zeta^{(\omega)}$ muss folglich von $L_{m-2}^{\prime(2)} \times L_{n-2}^{\prime(3)}$ abhängen. Aus (3) erhält man also

$$
E^{\prime(3)}(\xi .)=D^{(3)}\left(C^{(2)}(\xi)\right) \text {. }
$$

Genav so beweist man

$$
E^{(2)}(\zeta)=C^{(1)}\left(D^{(g)}(\zeta)\right)
$$

für einen allgemeinen Punkt $\zeta$ in $\Gamma_{3} \bullet E^{\prime}=E$ genügt also der Bedingung (1). Zusammenfassend haben wir

Satz 2. Für beliebige Korrespondenzen $C, D$ auf $\Gamma_{12}$ bzw. $\Gamma_{23}$ gibt es eine und nur eine Korrespondenz $E$ auf $\Gamma_{13}$, so dass es für beliebige Punkte $a, c$ in $\Gamma_{1}$ bzw. $\Gamma_{3}$

$$
E^{(3)}(a)=D^{(3)}\left(C^{(2)}(\alpha)\right), \quad E^{(1)}(c)=C^{(1)}\left(D^{(2)}(c)\right)
$$

gilt. Wir nennen $E$ das Produkt von $C$ und $D$ :

$$
E=C \times D \text {. }
$$

Wenn eines von $C, D$ nur aus ausgearteten irreduziblen Korrespondenzen besteht, so gilt dasselbe anch für $E=C \times D$ und wenn $C$ und $D$ keine ausgeartete Korrespondenz enthalten, so enthält auch $E=C \times D$ keine solche Korrespondenz.

Nun bildet bekanntlich die Gesamtheit aller Korrespondenzen auf $\Gamma_{12}=\Gamma_{1}$ $\times \Gamma_{2}$ einen $\cdot$ Modul, den $\cdot$ Korrespondenzenmodul $\mathfrak{E}\left(\Gamma_{1}, \Gamma_{2}\right)$ von $\Gamma_{1}, \Gamma_{2} \cdot$ In $\mathbb{C}\left(\Gamma_{1}, \Gamma_{2}\right)$ erzeugen die Korrespondenzen der Form $(a) \times \Gamma_{2}$ bzw. $\Gamma_{1} \times(b)$ je 
einen Teilmodul $\mathfrak{A}_{1}\left(\Gamma_{1}, \Gamma_{2}\right)$ bzw. $\mathfrak{A}_{2}\left(\Gamma_{1}, \Gamma_{2}^{\prime}\right)$. Die Summe von $\mathfrak{A}_{1}\left(\Gamma_{1}, \Gamma_{2}\right)$, $\mathfrak{U}_{2}\left(\Gamma_{1}, \Gamma_{2}\right)$ bezeichnen wir mit $\mathfrak{A}\left(\Gamma_{1}, \Gamma_{2}\right)$. Ebenso erzeugen alle nicht ausgeartete irreduzible Korrespondenzen einen Teilmodul $\odot\left(\Gamma_{1}, \Gamma_{2}\right)$. Es gilt offenbar die Zerlegungen in direkte Summen:

$$
\begin{aligned}
& \mathfrak{C}\left(\Gamma_{1}, \Gamma_{2}\right)=\mathfrak{G}\left(\Gamma_{1}, \Gamma_{2}\right)+\mathfrak{A}\left(\Gamma_{1}, \Gamma_{2}\right), \\
& \mathfrak{U}\left(\Gamma_{1}, \Gamma_{2}\right)=\mathfrak{A}\left(\Gamma_{1} \Gamma_{2}\right)+\mathfrak{U} \mathfrak{Z}\left(\Gamma_{1}, \Gamma_{2}\right) .
\end{aligned}
$$

Diese Moduln haben ersichtlich birational invariante Bedeutungen, d.h. das Bild von einem solchen Moduln, z.B. das von $\mathfrak{C}\left(\Gamma_{1}, \Gamma_{2}\right)$ bei einer birationalen 'Transformation, welche $\Gamma_{12}=\Gamma_{1} \times \boldsymbol{\Gamma}_{2}$ auf $\Gamma_{12}^{\prime}=\Gamma_{1}^{\prime} \times \Gamma_{2}^{\prime}$ abbildet, fällt mit $\tilde{\mathbb{C}}\left(\Gamma_{1}^{\prime}, \Gamma_{2}^{\prime \prime}\right)$ zusammen. Wir bezeichnen ferner den von $\mathfrak{I}$ und von der Korrespondenzen der Form $\left[\Gamma_{12}, f\left(x, y, u^{\prime}\right)\right]\left(f\left(x, y, u^{\prime}\right)=\right.$ beliebige Form über $\left.k\right)$ erzeugten Teilmodul mit $\mathfrak{R}\left(\Gamma_{1}, \Gamma_{2}\right)$ und den Restlklassenmodul $\mathbb{C}\left(\Gamma_{1}, \Gamma_{2}\right)$ / $\mathfrak{A}\left(\Gamma_{1}, \Gamma_{2}\right)$ mit $\mathbb{M}\left(\Gamma_{1}, \Gamma_{2}\right)$. $\mathbb{N}\left(\Gamma_{1}, \Gamma_{2}\right)$ nennt man den Multiplikatorenmodul von $\Gamma_{3}, \Gamma_{2}$. Dass $\mathfrak{N}\left(\Gamma_{1}^{\prime}, \Gamma_{2}^{\prime}\right)$ und somit auch $\mathfrak{D}\left(\Gamma_{1}, \Gamma_{2}\right)$ birational invariante Moduln sind, sieht man unmittelbar aus Satz 6 in I ein.

Späterer Anwendung wegen schalten wir hier einige Hilfssätze ein.

Hilfssatz 1. In jeder Restklasse von $\mathfrak{I}\left(\Gamma_{1}, \Gamma_{2}\right)$ gibt es eine solche Korrespoodenz

$$
C=\sum_{i=1}^{\alpha} C_{i}
$$

welche folgende Bedingungen erfüllt:

i) $C_{i}$ sind von einander verschieden,

ii) der allgemeine Punkt $\left(\xi^{(i)}, \eta^{(i)}\right)$ von $C_{i}$ ist so beschaffen, dass $k\left(\xi^{(i)}, \eta^{(i)}\right)$ $\mid k\left(\xi^{(i)}\right)$ separabel ist,

iii) $C_{i}(i=1, \ldots, \alpha)$ fallen mit keiner von den von vornherein angegebenen endlichvielen irreduziblen Korrespondenzen $D_{j}(j=1, \ldots, \beta)$ zusammen: $C_{i} \neq D_{j}(i=1, \ldots, \alpha, j=1, \ldots, \beta)$.

Beweis. Es sei $C^{*}$ eine beliebige Korrespondenz in einer Restklasse von $\mathfrak{M}\left(\Gamma_{1}, \Gamma_{2}\right)$ und $\xi$ ein allgemeiner Funkt von $\Gamma_{1}$. Wir wählen eine Punktgruppe $G=\left\{b^{(\rho)} ; \rho=1, \ldots, \gamma\right\}$ in $\Gamma_{2}$ mit genügend grosser Ordnung $\gamma$. Wir nehmen an dass jedes $b^{(P)}$ in $k$ liegt und dass die Ordnung $\delta$ von $\mathrm{G}+C^{*(2)}(\xi)$ zu $p$ prim ist. Wir bezeichnen mit $A_{\lambda}$ die allgemeine Punktgruppe der Vollschar der mit $G+C^{*(2)}(\xi)$ äquivalenten effektiven Divisoren und setzen

$$
A_{\lambda}+B=\left[\Gamma_{2}, \lambda_{0} g_{0}(y)+\lambda_{1} g_{1}(y)+\ldots+\lambda_{r} g_{r}(y)\right],
$$

wobei $B$ einen geeigneten festen Divisor und $g_{i}(y)$ Formen in $k(\xi)[y]$ bedeuten. Wenn $\gamma$ genügend gross gewählt ist, so folgt nach dem Riemann-Rochschen Satz, 
rass $A_{\lambda}$ genau der von $\lambda$ abhängige Teil von $\left[\Gamma_{2}, \Sigma \lambda_{i} g_{i}(y)\right]$ ist. Nach dem verallgemeinerten Satz von Bertini ${ }^{6)}$ sind also die Punkte $\eta^{(\mu)}(\mu=1, \ldots, \delta)$ ron $A_{\lambda}$ einander konjugiert über $k(\xi, \lambda)$ und fallen je $p^{e}$ zusammen, falls der Exponent von $k\left(\xi, \lambda, \eta^{(1)}\right), / k(\xi, \lambda) p^{e}$ ist. Da aber $\delta z u p$ prim ist, so folgt $p^{e}=1$, d.h. dass $\boldsymbol{\eta}^{(\mu)}$ alle voneinander verschieden sind. Durch geeignete Spezialisierung $\lambda \rightarrow \lambda^{\prime}\left(\lambda^{\prime} \epsilon k(\xi)\right)$ kann man also eine Punktgruppe $A_{\lambda}$, erhaiten, welche aus voneinander verschicdenen, über $k(\xi)$ algebraischen Punkten $\eta^{(\mu)}(\mu=1, \ldots, \delta)$ besteht. $1) a$

$$
G+C^{*(2)}(\xi) \equiv A_{\lambda}{ }^{7}
$$

ist, gibt es andereseits Formen $\varphi_{1}(y), \varphi_{2}(y)$ von demselben Grad in $k(\xi)[y]$, so dass

$$
\left[\Gamma_{:}, \varphi_{1}(y)\right]+G+C^{*(2)}(\xi)=\left[\Gamma_{:}, \varphi_{2}(y)\right]+A_{\lambda^{\prime}}
$$

gilt. Folglich gibt es zwei Formen $\phi_{3}(x, y), \Phi_{2}(x, y)$ in $k[x, y]$, welche dieselken Grade in $x$ und $y$ besitzen und

$$
\frac{\Phi_{1}(\xi, y)}{\Phi_{2}(\xi, y)}=\frac{\varphi_{1}(y)}{\varphi_{2}(y)},\left[\Gamma_{2}, \Phi_{1}(\xi, y)\right]+G+C^{*(2)}(\xi)=\left[\Gamma_{2}, \Phi_{2}(\xi, y)\right]+\Lambda_{\lambda^{\prime}}
$$

erfüllen. Setzt man also

$$
C=C^{*}+\sum_{p=1}^{\gamma} \Gamma_{1} \times\left(b^{(p)}\right)+\left[\Gamma_{12}, \Phi_{1}(x, y)\right]-\left[\Gamma_{12}, \Phi_{2}(x, y)\right],
$$

so liegt $C$ in derselben Restklasse in $\operatorname{SA}\left(\Gamma_{1}, \Gamma_{2}\right)$ wie $C^{*}$ und es gilt

$$
C^{(2)}(\xi)=A_{\lambda^{\prime}}=\left\{\eta^{(\mu)} ; \mu=1, \ldots, \delta\right\} .
$$

Lässt man aus $C$ irreduzible Korrespondenzen in $\mathfrak{A}_{1}\left(\Gamma_{1}, \Gamma_{2}\right)$ weg und bezeichnet den Rest wieder mit $C$, so bleibt (4) noch gültig. Da aber $\eta^{(\mu)}$ alle voneinander verschieden sind, so folgt hieraus, dass die Bedingungen i), ii) erfüllt sind. Man bemerke dabei, dass ein $\left(\xi, \eta^{(\mu)}\right)$ einen allgemeinen Punkt einer in $C$ enthaltenen irreduziblen Korrespondenz darstellt. Spezialisiert man $\eta^{(\mu)}$ ausserdem so, dass $\eta^{(\mu)}$ mit keinem Punkt von $D_{j}^{(2)}(\xi)$ zusammenfällt, so wird auch die Bedingung iii) erfüllt. ${ }^{8)}$

Hilfssatz 2. Es sei $C$ bzw. $D$ eine Korrespondenz auf $\Gamma_{12}$ bzw. $\Gamma_{2: 3}$. Wenn $C$ in $\mathfrak{N}\left(\Gamma_{1}, \Gamma_{2}\right)$ oder $D$ in $\mathfrak{N}\left(\Gamma_{2}, \Gamma_{3}\right)$ liegt, so liegt $C \times D$ in $\mathfrak{N}\left(\Gamma_{1}, \Gamma_{3}\right)$.

Beweis. Man hat offenbar den Satz nur in dem Fall zu beweisen, wo $C$ eine nicht ausgeartete irreduzible Korresponden $z$ auf $\Gamma_{12}$ ist und $D=\left[\Gamma_{23}, g\left(y, z, v^{\prime}\right)\right]$ mit einer geeigneten Form $g\left(y, z, v^{\prime}\right)$ gilt. Es sei $(\xi, \eta)$ ein allgemeiner Punkt

6) Vgl. 1.c. 5).

7) $G \equiv F$ bedeutet, dass die Punktgruppen $G, F$ äquivalent sind.

8) Dieser Hilfssatz lässt sich auch arithmetisch bewiesen, vgl. M. Deuring, Arithmetische Theorie der Korrespondenzen algebraischer Funktionenkörper II, Crelle's Jour. 183 (1.940). Siehe auch eine spätere Abhandlung des Verfassers. 
von $C$ und

$$
C^{(2)}(\xi)=\left\{\eta^{(p)}\right\}, \eta^{(1)}=\eta
$$

$F(\xi, z)=I_{\rho} g\left(\eta^{(\rho)}, z, v^{\prime}\right)$ ist dann offenbar eine Form in $k(\xi)[z]$. Durch geeignete Normierung von $\eta^{(p)}$ kann man sogar erreichen, dass es in $k[\xi, z]$ liegt. Wie oben oft gezeigt worden ist, gilt dann für $E=\left[\Gamma_{13}, F(x, z)\right]$

$$
E^{(3)}(\xi)=D^{(3)}\left(C^{(2)}(\xi)\right)=(C \times D)^{(3)}(\xi) \text {. }
$$

Nach Satz 1 ist also

$$
E \equiv C \times D \quad \bmod . \mathfrak{A}_{1}\left(\Gamma_{1}, \Gamma_{: 3}\right) .
$$

Aus $E \in \mathbb{R}\left(\Gamma_{1}, \Gamma_{3}\right)$ folgt dann $C \times D \in \mathbb{R}\left(\Gamma_{1}, \Gamma_{3}\right)$, wie behauptet wurde.

Wir beweisen nun den folgenden wichtigen

Satz $3^{\prime)}$. Eine Korrespondenz $C$ auf $\Gamma_{12}$ liegt dann und nur dann in $\mathfrak{R}\left(\Gamma_{1}\right.$, $\left.\Gamma_{2}\right)$, wenn für beliebige Punkte $a, \alpha^{\prime}$ in $\Gamma_{1}^{\prime}$ immer

$$
C^{(2)}(a) \equiv C^{(2)}\left(a^{\prime}\right)
$$

gilt. Wan sagt in diesem Fall, dass $C$ die Wertigkeit Null besitzt ${ }^{10)}$. Es gilt dann für beliebige Punkte $b, b^{\prime}$ in $\Gamma_{2}$ immer

$$
C^{(1)}(b) \equiv C^{(1)}\left(b^{\prime}\right)
$$

Beweis. Wenn $C$ in $\mathfrak{A}\left(\Gamma_{1}, \Gamma_{2}\right)$ liegt, so gilt ofienbar für beliebige Punkts $a, a^{\prime}$ in $\Gamma_{1}^{\prime}$

$$
C^{(2)}(a)=C^{(2)}\left(a^{\prime}\right)
$$

und wenn $C=\left[\Gamma_{12}, f\left(x, y, u^{\prime}\right)\right]$ ist, so ist

$$
C^{(2)}(a)=\left[\Gamma_{2}, f\left(a, y, u^{\prime}\right) \equiv\left[\Gamma_{2}, f\left(a^{\prime}, y, u^{\prime}\right)\right]=C^{(2)}\left(a^{\prime}\right)\right.
$$

Daraus folgt, dass (5) für eine beliebige Korrespondenz in $\mathfrak{N}\left(\Gamma_{1}, \Gamma_{2}\right)$ gilt. Nun sei umgekehrt $C$ eine Korrespondenz in $\widetilde{C}\left(\Gamma_{1}, \Gamma_{2}\right)$, für welche (5) gilt. Nach dem eben Bewiesenen kann man $C$ durch eine andere Korrespondenz ersetzen, welche in der Restklasse von $\mathrm{C}$ in $\mathfrak{M}\left(\Gamma_{1}, \Gamma_{2}\right)$ liegt, ohne die Gültigkeit von (5) zu verletzen. Nach Hílfssatz 1 können wir also annehmen, dass $C$ die Bedingungen i), ii) in demselben Hilfssatz erfüllt. Nun gibt es nach (5) eine Schar $G(\lambda, y)=\lambda_{0} g_{0}(y)+\lambda_{1} g_{1}(y)+\ldots+\lambda_{r} g_{r}(y)$ über $k$, so dass für einen beliebigen in $k$ liegenden Punkt $a$ auf $\Gamma_{1}$ immer

$$
C^{(2)}(a)+B=\left[\Gamma_{2}, G(\lambda(a), y)\right], B=\left\{b^{(\mu)} ; \mu=1, \ldots, \beta\right\}
$$

9) Dieser Satz entspricht Satz 8, $\overline{8}$ in M. Deuring, Arithmetische Theorie der Korrespondenzen algebraischer Funktionenkorper I, Crelle's Jour. 177 (1937). Deuring hat diesen Satz aus seiner Sätzen 7, 7 abgeleitet, welche eine Verallgemeinerung des lissischen Additionstheorems (nebst Umkehrung) bilden. Man siehr aber leicht, dass auch umgekelırt das Additionstheorem nebst Umkehrung (Satz 7, 7) sich aus unserem Satz 3 unmittelbar ableiten lässt. Der Zusammenhang der Denringscben arithmetischen Theorie mit unserer geometrischen Theorie soll in einer späteren Abhandlung ausführlich dargestellt werden.

10) Vgl. z.B. Severi-Löffler, Vorlesungen über algebraische Geometrie, Berlin (1921), S. 162. Allgemeine Weitigkeitskorrespondenzen werden wir nachher untersuchen. 
gilt, wobei $\lambda(a)$ eine geeıgnete Spezialisierung von $\lambda$ und $B$ eine feste Punktgruppe in $k$ ist. Da aber die Dimension einer Vollscher bei der Körpererweiterung $k(\xi) / k$ des Grundkörpers $k$ invariant bleibt, so gilt (7) anch für einen allgemeinen Punkt $\xi$ von $\Gamma_{1}$. Setzt man also

$$
C^{(2)}(\xi)=\left\{\eta^{(\rho)} ; \rho=1, \ldots, \alpha\right\},
$$

so besitzt das lineare Gleichungssystem

$$
\begin{gathered}
G\left(\lambda(\xi), \eta^{(\rho)}\right)=\lambda_{0}(\xi) g_{11}\left(\eta^{(\rho)}\right)+\lambda_{1}(\xi) g_{1}\left(\eta^{(P)}\right)+\ldots+\lambda_{r}(\xi) g_{r}\left(\eta^{(\rho)}\right), \\
\rho=1, \ldots, \alpha
\end{gathered}
$$

mindestens eine nicht triviale Lösung $\lambda(\xi)=\left(\lambda_{0}(\xi), \lambda_{i}(\xi), \ldots, \lambda_{r}(\xi)\right)$ in $K=$ $k\left(\xi, \eta^{(1)}, \ldots, \eta^{(\alpha)}\right)$. Nach Voraussetzung ist $K$ eine separable galoissche Erweiterung von $k(\xi)$ und das Gleichungssystem (8) ist ersichtlich invariant gegenüber den Galoisautomorphismen von $K / k(\xi)$. Durch Spurbildung kann man also eine Lösung in $k(\xi)$ erhalten. Es gibt also Formen $\lambda(x)=\left(\lambda_{0}(x), \lambda_{1}(x), \ldots\right.$, $\left.\lambda_{r}(x)\right)$ in. $k[x]$, so dass $G(\lambda(x), y)$ für $x=\xi, y=\eta^{(p)}$ verschwindet und

$$
C^{(2)}(\xi)+D^{(2)}(\xi)=\left[\Gamma_{2}, G(\lambda(\xi), y)\right] \text { mit } D=\Sigma \Gamma_{1} \times\left(b^{(\mu)}\right)
$$

gilt. Daraus sieht man sofort, dass $C$ mit $\left[\Gamma_{12}, G(\lambda(x), y)\right]$ bis auf Korrespondenzen in $\mathfrak{A}\left(\Gamma_{1}, \Gamma_{2}\right)$ übereinstimmt, folglich in $\mathfrak{N}\left(\Gamma_{1}, \Gamma_{2}\right)$ ljegt, w.z.b.w.

Nun seien $\Gamma_{1}, \Gamma_{2}$ birational äquivalent. Wir können also annehmen,

$$
P_{l}=P_{m}, \Gamma_{1}=\Gamma_{2}=\Gamma \text {. }
$$

Wendet man nun Satz 2 auf $\Gamma_{1}=\Gamma_{2}=\Gamma_{3}=\Gamma$ an. so sieht man sofort, dass $\mathfrak{C}(\Gamma, \Gamma)$ ir diesem Fall einen Ring bildet: den "Korrespondenzenring " $\mathfrak{C}(\Gamma)$ von $\Gamma$. Es ist auch leicht zu sehen, dass $\cong(\Gamma)=\Im(\Gamma, \Gamma)$ einen Teilring und $\mathfrak{A}(\Gamma)=\mathfrak{U}\left(\Gamma_{1} \Gamma\right), \quad \mathfrak{A}_{1}(\Gamma)=\mathfrak{U}_{1}\left(\Gamma_{1} \Gamma\right), \mathfrak{A}_{2}\left(\Gamma^{\prime}\right)=\mathfrak{U}_{2}\left(\Gamma_{1} \Gamma\right)$ sogar Ideale in $\mathfrak{C}(\Gamma)$ bilden. Es gilt dann z.B.

$$
\mathfrak{C}(\Gamma) / \mathfrak{A}(\dot{\Gamma}) \cong \mathscr{S}(\Gamma) \text {. }
$$

Aus Hilfssatz 2 folgt ferner, dass $\mathfrak{N}(\Gamma)=\Re\left(\Gamma, \Gamma^{\top}\right)$ auch ein Ideal von $\mathbb{C}(\Gamma)$. ist. Der Restklassenring $\mathfrak{M}(\Gamma)=\mathbb{M}(\Gamma, \Gamma)$ nach diesem Ideal nennt mar den “Multiplikatorenring" von $\Gamma \cdot \operatorname{PA}(\Gamma)$ oder allgemeiner $\mathfrak{M}\left(\Gamma_{1}, \Gamma_{2}\right)$ bildet eine Verallgemeinerung des Rings oder Moduls der klassischen komplexen Multiplikationen von $\Gamma$ oder von $\Gamma_{1}, \Gamma_{2}{ }^{11}$. Die Struktur von $\mathfrak{M}(\Gamma)$ sijll in einer späteren Arbeit ausführlich untersucht werden.

11) Vgl. M. Deuring, l.c. 9) 